\title{
An economic decision-making support system for selection of reproductive management programs on dairy farms
}

\author{
J. O. Giordano, P. M. Fricke, M. C. Wiltbank, and V. E. Cabrera ${ }^{1}$ \\ Department of Dairy Science, University of Wisconsin, Madison 53706
}

\begin{abstract}
Because the reproductive performance of lactating dairy cows influences the profitability of dairy operations, predicting the future reproductive and economic performance of dairy herds through decision support systems would be valuable to dairy producers and consultants. In this study, we present a highly adaptable tool created based on a mathematical model combining Markov chain simulation with partial budgeting to obtain the net present value (NPV; $\$ /$ cow per year) of different reproductive management programs. The growing complexity of reproductive programs used by dairy farms demands that new decision support systems precisely reflect the events that occur on the farm. Therefore, the model requires productive, reproductive, and economic input data used for simulation of farm conditions to account for all factors related to reproductive management that increase costs and generate revenue. The economic performance of 3 different reproductive programs can be simultaneously compared with the current model. A program utilizing $100 \%$ visual estrous detection (ED) for artificial insemination (AI) is used as a baseline for comparison with 2 other programs that may include $100 \%$ timed AI (TAI) as well as any combination of TAI and ED. A case study is presented in which the model was used to compare 3 different reproductive management strategies (100\% ED baseline compared with two 100\% TAI options) using data from a commercial farm in Wisconsin. Sensitivity analysis was then used to assess the effect of varying specific reproductive parameters on the NPV. Under the simulated conditions of the case study, the model indicated that the two 100\% TAI programs were superior to the $100 \%$ ED program and, of the $100 \%$ TAI programs, the one with the higher conception rate (CR) for resynchronized AI services was economically superior despite having higher costs and a longer interbreeding interval. A $4 \%$ increase in $\mathrm{CR}$ for resynchronized AI was sufficient for the inferior
\end{abstract}

Received March 21, 2011.

Accepted August 9, 2011.

${ }^{1}$ Corresponding author: vcabrera@wisc.edu
$100 \%$ TAI to outperform the superior program. Adding ED to the $100 \%$ TAI programs was only beneficial for the program with the lower $\mathrm{CR}$. The improvement in service rate required for the $100 \% \mathrm{ED}$ program to have the same NPV as the superior $100 \%$ TAI program was $12 \%$. The decision support system developed in this study is a valuable tool that may be used to assist dairy producers and industry consultants in selecting the best farm-specific reproductive management strategy.

Key words: economics, reproductive program, dairy farm

\section{INTRODUCTION}

Reproductive performance of dairy herds has long been recognized as a major contributor to the overall profitability of dairy operations (Louca and Legates, 1968; Oltenacu et al., 1981; Britt, 1985). Total milk sales, number of culled cows, and number of replacement heifers born are among the major sources of income affected by reproductive performance of lactating dairy cows (Britt, 1985; De Vries, 2004; Meadows et al., 2005). Feed cost, labor cost, and veterinary expenses are also highly influenced by the reproductive program because lactation length, dry period length, and number of services to conceive are highly correlated with the reproductive performance of cows. Therefore, at the time of selecting a long-term reproductive management strategy, all factors that contribute to the variation in reproductive and economic performance need to be considered for analysis.

Recent advances in the understanding of reproductive physiology of lactating dairy cows has led to the development of numerous management strategies and technologies aimed to improve overall reproductive efficiency of dairy herds (Thatcher et al., 2006). Estrous detection aids (Xu et al., 1998; Rorie et al., 2002), hormonal synchronization of estrus (Folman et al., 1984; Momcilovic et al., 1998), timed artificial insemination (TAI; Pursley et al., 1995; Moreira et al., 2001; Souza et al., 2008), and computerized record systems (Losinger and Heinrichs, 1996) are among the technologies most commonly used by modern dairy farms to improve breeding efficiency and fertility (Caraviello et 
al., 2006). Unfortunately, under most circumstances, it is difficult to determine whether the on-farm application of these new reproductive management programs and technologies will translate into actual economic benefits to the farm. Assessing the economic impact of different reproductive management strategies under the commercial conditions imposed on a dairy farm represents a major challenge due to the nature of the dairy cow productive cycle, which is characterized by a significant lag time between changes in reproductive performance and the realization of economic benefits or losses. Because of the complex interplay between numerous factors that may affect reproduction and the wide variability observed across dairy operations, the approach to assess the economics of reproduction must be inclusive and flexible. Attempts have been made to measure the economic impact of different reproductive programs under experimental conditions (Britt and Gaska, 1998; Arbel et al., 2001; Tenhagen et al., 2004; Lima et al., 2010). Performing this type of comparison routinely, however, may not be feasible for commercial dairy operations due to the difficulty and inconvenience of simultaneously applying more than one reproductive program on a farm. Experimental tests in management programs that may lead to changes in reproductive performance could also negatively affect profitability of the dairy operation.

Another approach used to overcome the limitations of economic-based analysis of reproduction on dairy farms has been to develop simulation models using multiple computational methods. For example, the OptiCow model (Groenendaal et al., 2004) applied the net revenue technique to determine optimal breeding and replacement decisions for dairy herds. Using dynamic programming, De Vries (2004) created the DairyVIP model to assess the economics of delayed replacement when performance is seasonal and then calculated the value of a pregnancy in dairy cattle (De Vries, 2006). More recently, Olynk and Wolf (2009), using first- and second-degree stochastic dominance, determined the most economically optimal program from a set of reproductive management scenarios that used either visual detection of estrus or synchronization of ovulation while also considering human factors such as risk aversion. Although results are based on simulations of multiple events, the development of support systems from these types of models greatly benefit the dairy industry because they can be used to assist producers and consultants in the decision-making process at the time of selecting the best reproductive management program for a specific dairy farm.

Because of the growing complexity of reproductive management programs used by dairy farms, it is critical that new decision support systems precisely reflect the events that occur at the farm level after the application of a specific management program. Models become more reliable in predicting the future behavior of the system when the number of assumptions used is minimized and all sources of cost and revenue that may affect the economics of the operation are taken into account. Because the specific management strategies and conditions of a particular dairy farm affect the amount of resources and time used to perform a reproductive management program, different labor efficiencies, total cost, and results will be observed. Therefore, at the time of making a comprehensive assessment for a dairy, it is critical to include in the analysis data generated from the farm under consideration. This is particularly important at the time of the decision to adopt a particular reproductive management program because of the lag time between the moment to invest resources to perform a program, the timing to achieve better reproductive performance, and the even longer period until economic benefits are realized.

The specific objectives of this study were (1) to develop a robust yet user-friendly decision-making support system by combining a sequential reproductive Markov chain simulation model with partial budgeting to estimate potential differences in profitability when applying different reproductive management strategies on a specific dairy farm; and (2) use the model to compare the economic outcomes of 3 reproductive management strategies for a specific dairy farm and subsequently perform sensitivity analysis to assess the effect of varying specific reproductive parameters in the decision-making process.

\section{MATERIALS AND METHODS}

\section{Mathematical Simulation Model and Software Tool}

A simulation model was created based on the integration of multiple mathematical equations. A Markov chain simulation framework was used to calculate herd dynamics and integrated with multiple partial budgets to estimate the net present value (NPV) of 3 different reproductive management programs to be compared simultaneously. The mathematical model was then used to develop a software decision support tool consisting of multiple modules, including lactation, reproductive performance, culling, AI service cost, expected monetary value (EMV), and reproductive parameters defined in Excel for Windows (version 2010, Microsoft, Redmond, WA). Each module contains specific predefined equations used to perform calculations with the input data. The user interface consists of an input and a result sheets. The input sheet is used to enter specific farm productive, economic, and reproductive parameters, 
whereas the result sheet displays calculations results based upon the input parameters after performing the simulations. This model was used for the case study presented in this paper.

\section{Calculation of the NPV of Reproductive Programs}

The daily NPV (\$/cow per day) of a specific reproductive program during a lactation is defined as the discounted EMV (DEMV) of the proportion of cows becoming pregnant in consecutive reproductive services until a defined DIM plus the DEMV of that proportion of cows not becoming pregnant until the defined DIM:

$$
N P V_{r, S}=D E M V(P)_{S}+D E M V(N P)_{S},
$$

where $r=$ the reproductive program (e.g., PresynchOvsynch followed by Ovsynch with estrous breeding in between); $S=$ number of reproductive services within defined DIM [e.g., 5 services at 250 DIM for a Presynch-Ovsynch program with a voluntary waiting period $(\mathbf{V W P})=55 \mathrm{~d}$ and interval between resynchronizations $=42 \mathrm{~d}], P=$ proportion of pregnant cows, and $N P=$ proportion of nonpregnant cows $=1-P$.

The DEMV for cows becoming pregnant $(P)$ is calculated as the sum of the DEMV incurred in consecutive reproductive services. For each service, this includes the difference between the expected monetary value and the specific costs of the reproductive program:

$$
\operatorname{DEMV}(P)_{S}=\sum_{s=1}^{S} \delta\left(P_{s}\right)\left[\operatorname{EMV}(P)_{s}-C S_{s}\right],
$$

where $\delta=$ daily discount rate, $s=$ reproductive service, $E M V(P)=$ EMV for cows becoming pregnant, and $C S$ $=$ cost of reproductive service.

The DEMV for nonpregnant $(N P)$ cows is calculated as the DEMV incurred for cows not becoming pregnant after the last service within the defined DIM plus the cost of their replacement (culled for reproductive failure).

To perform equivalent calculations between $P$ and $N P$ cows and to compare them under similar timeline, costs and benefits for $N P$ cows were prorated to an entire lactation, including an assumed gestation period as if the $N P$ cows would have started a pregnancy at the defined DIM:

$$
\begin{gathered}
D E M V(N P)_{S}=\delta(N P)_{S}\left[E M V(N P)_{S}\right. \\
\left.+(S V+M V C-H R V) /\left(D O_{S}+G\right)\right]
\end{gathered}
$$

where $E M V(N P)=$ EMV for cows not becoming pregnant, $S V=$ salvage value of a cow, $M V C=$ market value of a calf (weighted average of male and female offspring, and stillbirth), $H R V=$ heifer replacement value, $D O=$ days open (d), and $G=$ gestation length (d).

The EMV for a defined service for cows not becoming pregnant was calculated based on the value of the milk produced, the feed cost during lactation, the cost associated with involuntary culling, and the cost associated with unexpected death until the defined DIM [Eq. 4], whereas the EMV for a defined service for cows becoming pregnant was calculated including all the factors of nonpregnant cows plus the value of a newborn and the cost of feed for the dry period [Eq. 5]:

$$
\begin{gathered}
E M V(N P)_{S}=[M P V(N P)-C F M(N P) \\
-C C(N P)-C D(N P)], \\
E M V(P)_{s}=\left[M P V(P)_{s}+V N B_{s}-C F M(P)_{s}\right. \\
\left.-C F D_{s}-C C(P)_{s}-C D(P)_{s}\right],
\end{gathered}
$$

where $M P V=$ milk production value $(\$ / \mathrm{d})$ :

$$
\begin{aligned}
M P V(N P)_{S} & =\sum_{D I M=1}^{D O_{S}} M P_{S, L} \times P M /\left(D O_{S}+G\right) \text { and }[6] \\
M P V(P)_{s} & =\sum_{D I M=1}^{D O_{s}+G-D P} M P_{s, L} \times P M /\left(D O_{s}+G\right), \quad[7]
\end{aligned}
$$

where $M P=$ milk production $(\mathrm{kg}), L=$ parity (primiparous or multiparous), $D P=$ dry period (d), $P M$ $=$ price of milk $(\$ / \mathrm{kg}), V N B=$ value of a newborn of pregnant cow $(\$ / \mathrm{d})$, and $V N B_{s}=M V C /\left(D O_{s}+G\right),[8]$, where $M V C=$ market value of a calf, $M V C=[(M C V)$ $\times(M C P)+(F C V) \times(F C P)](1-S B),[9]$, where $M C V$ $=$ male calf value, $M C P=$ male calf proportion, $F C V$ $=$ female calf value, $F C P=$ female calf proportion, and $S B=$ proportion of stillbirth.

The male to female ratio for the $M V C$ calculations was set at 53.3:46.7 as reported in Silva del Rio et al. (2007), and $C F M=$ cost of feed for milking cows $(\$ / d)$ :

$$
\begin{aligned}
C F M(N P)_{S} & =\sum_{D I M=1}^{D O_{S}} F I_{S, L} \times F P /\left(D O_{S}+G\right) \text { and }[10] \\
C F M(P)_{s} & =\sum_{D I M=1}^{D O_{s}+G-D P} F I_{s, L} \times F P /\left(D O_{s}+G\right), \quad[11]
\end{aligned}
$$


where $F P=$ feed price $(\$ / \mathrm{kg}$ of $\mathrm{DM}), F I=$ feed ingested $(\mathrm{kg} /$ cow per day), $C F D=$ cost of feed for dry cows $(\$ / \mathrm{d})$, and $C F D_{s}=(D C F \times D P) /\left(D O_{s}+G\right)$, [12], where $D C F=$ daily cost of feed for dry cows $(\$ / \mathrm{d}), C C$ $=$ cost associated with involuntary culling,

$$
\begin{aligned}
C C(N P)_{S} & =\sum_{D I M=1}^{D O_{S}} R C_{S, L} \\
& \times(H R V-S V-V N B) /\left(D O_{S}+G\right) \text { and }
\end{aligned}
$$

$$
C C(P)_{s}=\sum_{D I M=1}^{D O_{s}+G} R C_{s, L} \times(H R V-S V-V N B) /\left(D O_{s}+G\right),
$$

where $R C=$ daily rate of culling, $C D=$ cost associated with unexpected death,

$$
\begin{aligned}
& C D(N P)_{S}=\sum_{D I M=1}^{D O_{S}} R D_{S, L} \times(H R V-V N B) /\left(D O_{S}+G\right) \text { and } \\
& {[15] } \\
& C D(P)_{s}= \sum_{D I M=1}^{D O_{s}+G} R D_{s, L} \times(H R V-V N B) /\left(D O_{s}+G\right),
\end{aligned}
$$

where $R D=$ daily rate of death.

An assumption of the model for the calculation of the NPV was that at the time a cow left the herd due to either involuntary culling, death, or failure to become pregnant within the specified DIM for breeding (reproductive failure), a new replacement heifer was available at the specified market value, which was charged as a cost for the reproductive program being analyzed. Likewise, the model assumed that all newborn calves from cows that became pregnant and those born from replacement heifers were sold at birth at their specific market value specified as an economic input variable.

\section{Calculation of the Survival Curves of Reproductive Programs}

Depending on the parameters of the reproductive program, the status of all cows was calculated in each service. This process estimated sequentially, as finite Markov chains, the percentage of cows eligible for breeding after the end of the VWP, the proportion of cows serviced, the percentage of cows becoming pregnant, and the percentage of cows not becoming pregnant on each service. The first state in the Markov chain process was represented by the nonpregnant cows, which could move to the next state of being inseminated following transition probabilities determined by the reproductive program. The probability of a cow being inseminated depended on the estrous detection rate for programs relying on estrous detection or the predefined timing of AI based on a synchronization of ovulation protocol. When the reproductive program combined both strategies (i.e., estrous detection and synchronization), a proportion of cows were considered inseminated after estrous detection $21 \mathrm{~d}$ after the previous $\mathrm{AI}$ and the remainder of the cows at the time of the predefined next TAI based on the synchronization protocol. Inseminated cows could then move to the pregnant state within the Markov chains following transition probabilities of $\mathrm{CR}$ defined by the reproductive program. After each reproductive event (AI), the proportion of cows failing to conceive, plus those not receiving an AI in that service attempt, returned to the nonpregnant state and were eligible for the next AI. This probabilistic process was endowed by the Markov property that a next state depends solely on the current state and the transition probabilities: $P_{s}=(C R)_{s} \times(S R)_{s}$, [17], where $C R=$ conception rate (number of pregnant cows per number of inseminated cows), $S R=$ service rate $=$ proportion of cows bred out from total number of breeding eligible after the end of the VWP, and $S R_{s}=B E_{s} \times B_{s},[18]$, where $B E=$ breeding eligible cows and $B=$ bred cows.

As defined by the reproductive program, breeding eligible cows $\left\{B E_{s}=\left(1-P_{s-1}\right)\right.$ and $\left.P_{0}=0,[19]\right\}$, are those nonpregnant (open) cows after the previous AI service. Inseminated cows are those receiving insemination after either estrous detection or completion of a synchronization protocol. The interbreeding interval (IBI) for synchronized services is defined by the protocol for $B E_{s}$.

The percentage of cows not becoming pregnant after a series of reproductive services for a defined reproductive program (reproductive services to defined cut-off DIM) is the complement of the aggregated proportion of cows becoming pregnant, which is calculated as the cumulative sum of pregnancies until a defined DIM:

$$
N P_{S}=1-\sum_{s=1}^{S} P_{s}
$$

The number of services to a defined cut-off DIM depends on the reproductive program. The last reproductive event occurs when DIM $\leq$ defined cut-off DIM.

\section{Calculated Reproductive Performance Parameters}

Based on the simulated herd dynamics from the reproductive performance entered with input data, the 
model calculates expected values for reproductive parameters typically used to monitor reproductive performance of dairy herds. Twenty-one-day pregnancy rate (21-d PR) indicates the rate at which eligible cows become pregnant (Ferguson and Galligan, 1999); it is calculated as the percentage of eligible cows that become pregnant during each 21-d period after the end of the VWP. The model also calculates 21-d service rate (21-d SR), which measures breeding efficiency or the ability of the farm to perform AI in eligible cows after either a detected estrus or TAI. Similar to 21-d PR, 21-d SR represents the percentage of eligible cows that receive AI during every 21-d period after the end of the VWP. Additional calculated parameters include average CR (number of cows pregnant over inseminated) for all breedings, days open (number of days from calving to conception for pregnant cows), and projected calving interval (PCI; actual interval between 2 calving events plus projected interval for nonpregnant cows, which is calculated by adding length of gestation to the cut-off DIM).

\section{Description of Productive, Reproductive, and Economic Parameters of the Model Within a Case Study}

The model allows for comparison of the economic performance of 3 reproductive management programs simultaneously. A program utilizing $100 \%$ estrous detection (ED) for AI is used as baseline for comparison with 2 other programs that may include 100\% TAI for first and subsequent services postpartum as well as any combination of TAI and ED. The synchronization programs for first-service TAI and resynchronization may differ and were selected from a list of available ovulation synchronization protocols for use on dairy farms. The reproductive performance of each management program under comparison was simulated by using user-defined parameters based on previous or future on-farm expected performance.

Reproductive Programs to Compare in Case Study. The economic and reproductive performance for 3 different reproductive management strategies (programs A through C) was simulated and compared using data from a high-producing commercial dairy farm in Wisconsin, where different ovulation synchronization protocols were tested during a period of 2 yr. Productive parameters were obtained from a DairyComp305 (Valley Agricultural Software, Tulare, CA) file from the dairy, and economic parameters were obtained from the Wisconsin Calculated Milk Cost of Production report for the November 2010 published by the Center of Dairy Profitability at the University of WisconsinMadison (http://cdp.wisc.edu/). General and specific reproductive parameters included in the experiment consisted of data collected from the DairyComp305 file and the results of an ovulation synchronization study conducted at the farm (Giordano et al., 2009).

Two of the programs compared (A and B) used synchronization of ovulation and TAI to perform AI services, whereas the third $(\mathrm{C})$ relied completely on ED to perform AI services. For reproductive programs A and B, all services were performed by TAI after synchronization of ovulation with specific protocols for first service and for second and subsequent AI services. Each week in programs A and B, all cows that were 56 \pm 3 DIM were enrolled in a Double-Ovsynch protocol (Souza et al., 2008) to receive their first service TAI at $82 \pm 3$ DIM. Briefly, the Double-Ovsynch protocol begins with a modified Ovsynch (Pre-Ovsynch; GnRH-7 $\left.\mathrm{d}-\mathrm{PGF}_{2 \alpha}-3 \mathrm{~d}-\mathrm{GnRH}\right)$ to presynchronize the estrous cycle and $7 \mathrm{~d}$ later, it continues with Breeding-Ovsynch (GnRH-7 d-PGF $\left.{ }_{2 \alpha}-56 \mathrm{~h}-\mathrm{GnRH}\right)$ followed by TAI 16 to $20 \mathrm{~h}$ after the last GnRH. For program A, second and subsequent AI services were performed after resynchronization of ovulation with an Ovsynch (Pursley et al., 1995) protocol ( $\left.\mathrm{GnRH}-7 \mathrm{~d}-\mathrm{PGF}_{2 \alpha}-56 \mathrm{~h}-\mathrm{GnRH}\right)$ initiated $32 \mathrm{~d}$ after a previous AI. This resynchronization program (usually referred to as D32 Resynch) began with cows receiving a $\mathrm{GnRH}$ injection regardless of pregnancy status $32 \mathrm{~d}$ after AI, and pregnancy diagnosis $7 \mathrm{~d}$ later by transrectal palpation of uterine contents; those cows diagnosed not pregnant continued the protocol by receiving $\mathrm{PGF}_{2 \alpha}$ and $\mathrm{GnRH} 56 \mathrm{~h}$ later. Between 16 to $20 \mathrm{~h}$ after the final $\mathrm{GnRH}$ injection, all cows received TAI. The resulting IBI (timeframe between 2 successive TAI breedings) for program A was $42 \mathrm{~d}$.

For program B, the second and subsequent AI services were performed after ovulation resynchronization with a Double-Ovsynch Resynch protocol similar to that described for first service. Cows enrolled in the Double-Ovsynch Resynch protocol (Pre-Resynch, GnRH-7 d-PGF $2 \alpha-3$ d-GnRH, 7 d later BreedingResynch, GnRH-7 d-PGF $\left.{ }_{2 \alpha}-56 \mathrm{~h}-\mathrm{GnRH}-16 \mathrm{~h}-\mathrm{TAI}\right)$ began the program by receiving a GnRH injection 22 d after a previous TAI regardless of their pregnancy status, 7 d later pregnancy diagnosis was performed by transrectal ultrasonography, and those cows found to be not pregnant continued the protocol to complete the presynchronization part of Double-Ovsynch by receiving $\mathrm{PGF}_{2 \alpha}$ and $\mathrm{GnRH} 72 \mathrm{~h}$ later. Seven days after completion of Pre-Resynch, all cows were enrolled in Breeding-Resynch and received TAI 16 to $20 \mathrm{~h}$ after the last GnRH injection of the Breeding-Resynch protocol. The resulting IBI for program B was $49 \mathrm{~d}$.

For program C (baseline), it was assumed that all cows were inseminated after visual observation of es- 
trus without aids. The VWP was set at 50 DIM and the duration of the estrous cycle was set at $21 \mathrm{~d}$, resulting in all second and subsequent AI services occurring at this time interval.

The maximum number of DIM until cows were inseminated (cut-off DIM) for the 3 programs was set at 330 because $95 \%$ of all AI services on this dairy occurred between the end of the VWP and this time point.

A detailed description of the 3 reproductive management programs compared is displayed in Table 1 . Programs A and B differed greatly from program C in that $100 \%$ of the services were performed on TAI after synchronization of ovulation, whereas program $\mathrm{C}$ relied solely on ED. As is commonly observed for dairy herds using $100 \% \mathrm{ED}$, the VWP was set at 50 DIM, whereas for the 2 programs using $100 \%$ TAI it was set at 82 DIM concurrent with the time of the first TAI service on this farm. The major differences between programs A and B were (1) the duration of the IBI, which was $42 \mathrm{~d}$ for the D32 Resynch protocol and $49 \mathrm{~d}$ for the Double-Ovsynch Resynch protocol, and (2) the different CR for the resynchronized TAI.

Cost of Reproductive Services for Synchronization Programs to Compare. The total cost of a first TAI, resynchronized TAI, and AI after estrus for each specific reproductive program was calculated. In the case of TAI services, the total cost was obtained by adding the cost of hormones administered for synchronization, labor required for hormone administration, AI (including labor and semen costs), and pregnancy diagnosis. Given that most reproductive programs use different protocols for first-service TAI and subsequent services (Resynch), the total cost per AI service was calculated similarly but individually for first TAI and Resynch: $C S_{s}=H O R_{s}+L A B_{s}+A I_{s}+P D_{s}$, [21], where $C S=$ total breeding cost (e.g., first postpartum
TAI or Resynch TAI), HOR $=$ hormones required for synchronization (\$/service), $L A B=$ labor required to administer hormone injections (\$/cow per service), $A I$ $=$ cost of insemination (includes semen and labor; $\$ /$ service), and $P D=$ pregnancy diagnosis $(\$ /$ cow per diagnosis).

To minimize the IBI, programs A and B used aggressive resynchronization strategies that required starting the Resynch protocol before the pregnancy status of previously inseminated cows was known. Consequently, cows that successfully conceived to a previous AI service received an extra $\mathrm{GnRH}$ injection in both programs. The model was capable of considering the cost associated with the administration of hormones for resynchronization to pregnant cows by calculating and adding this extra cost in the service attempt after which they conceived. This cost was calculated similarly to the cost of AI service except that charges for pregnancy diagnosis and AI were not included.

Cost of hormones for synchronization included all the necessary hormones administered to synchronize estrus or ovulation for TAI: $H O R_{s}=G n R H_{s}+(P G F)_{s}$ [22], where $\mathrm{GnRH}=$ total number of $\mathrm{GnRH}$ injections times the cost per dose $(\$)$ and $P G F=$ total number of $\mathrm{PGF}_{2 \alpha}$ injections times the cost per dose $(\$)$. The cost of GnRH and $\mathrm{PGF}_{2 \alpha}$ for the case study was set at $\$ 2.00$ and $\$ 2.50$ per dose, respectively, with the total number of injections of each hormone predetermined by the specific synchronization program (Table 2).

Labor cost associated with the administration of hormones for synchronization was calculated on a per cow per service basis (\$/cow per service). Each synchronization protocol required the administration of at least 2 hormone injections on specific days of the week according to the day in which cows were enrolled in the program. For programs A and B, Double-Ovsynch for first-service TAI began on Fridays, whereas D32

Table 1. Reproductive parameters for the 3 reproductive programs compared in the case study

\begin{tabular}{lccc}
\hline & \multicolumn{3}{c}{ Program $^{2}$} \\
\cline { 2 - 4 } Reproductive parameter & A & B & $\mathrm{C}^{3}$ \\
\hline Voluntary waiting period (d) & 82 & 82 & 50 \\
Interbreeding interval (d) & 42 & 49 & 21 \\
$\mathrm{CR}^{4}$ at first service postpartum (\%) & 30 & 45 & 33 \\
$\mathrm{CR}^{1}$ at second and later AI services (\%) & 39 & 30 \\
\hline${ }^{1}$ Reproductive parameters included in the case study consisted of data collected from a DairyComp305 (Valley \\
Agricultural Software, Tulare, CA) file and the results of a synchronization of ovulation study conducted at \\
the farm. \\
${ }^{2}$ Synchronization of ovulation was used for all AI breedings in programs A (Double-Ovsynch and D32 Resynch \\
at first and second or subsequent service, respectively) and B (Double-Ovsynch and Double-Ovsynch Resynch \\
at first and second or subsequent service, respectively), whereas AI was performed after estrous detection (ED) \\
in program C. \\
${ }^{3}$ The 21-d service rate for the ED program (C) was set at 50\%. \\
${ }^{4}$ CR = conception rate.
\end{tabular}


Table 2. Number of injections required to complete synchronization of ovulation protocols used in programs A and B for the case study

\begin{tabular}{llc}
\hline & \multicolumn{2}{c}{ Number of injections } \\
\cline { 2 - 3 } Synchronization protocol & GnRH & PGF $_{2 \alpha}$ \\
\hline First-service timed AI & & \\
Double-Ovsynch (A and B) & 4 & 2 \\
Resynchronization & 2 & 1 \\
D32 Resynch (A) & 4 & 2 \\
Double-Ovsynch Resynch (B) & & \\
\hline
\end{tabular}

Resynch in program A began on Mondays and DoubleOvsynch Resynch in program B began on Fridays. The total number of specific days of the week (e.g., number of Mondays) was predetermined by the synchronization protocol being applied:

$$
L A B_{s, d}=\sum_{d=1}^{7} C D_{s, d} N D_{s, d}
$$

where $d=$ day of the week (Sunday to Monday, 1 to 7 ), $C D=$ labor cost for day $d$, and $N D=$ number of days of the week (e.g., number of Mondays on which the cow will receive injections to complete the synchronization protocol).

Labor cost for a specific day of the week $d$ was obtained by multiplying the number of personnel involved on that day times the number of hours required to complete the injections times the cost per hour of labor. To obtain the cost per cow for a specific day $d$, the total labor cost for $d$ was divided by the total number of cows receiving injections on that specific day: $C D_{s, d}$ $=(P E R \times H \times C L A B)_{s, d} / C O W S_{s, d}[24]$, where $d=$ day of the week, $P E R=$ number of employees involved in administering the injections (no.), $H=$ number of hours (h), $C L A B=$ cost per hour of labor $(\$ / \mathrm{h})$, and $C O W S=$ number of cows injected.

Similar to the calculation of total cost of hormones, labor cost associated with hormone administration was added to the total cost for first-service TAI and for Resynch TAI. The ED programs did not incur costs for labor for hormone administration.

Labor cost for hormone administration was set at $\$ 15.00 / \mathrm{h}$ and was similar for the 2 programs involving synchronization. The number of laborers and number of hours per day of work for each program depended on the number of injections applied on any given day and the number of cows receiving injections.

Cost associated with AI was calculated by adding the average price of a semen unit and the cost of labor to perform an AI: $A I_{s}=S E M_{s}+C A I_{s}$, [25], where $S E M$ $=$ average cost of semen unit (\$/unit) and $C A I=$ labor cost for AI (\$/cow). The average cost of an AI breeding was set at $\$ 10.00$.
The model could calculate the cost of the first pregnancy diagnosis post AI for rectal palpation, ultrasonography, or blood testing. For rectal palpation and ultrasonography, the cost of pregnancy diagnosis for a given AI service was calculated by multiplying the number of hours necessary to diagnose all cows available for pregnancy diagnosis on a specific day by the cost per hour of labor. To obtain the cost per cow, the total cost for a specific day was divided by the number of cows diagnosed on that day: $P D_{s, d}=(H P D \times$ $C P D)_{s, d} / C O W S_{s, d}[26]$, where $H P D=$ number of hours required to complete the diagnosis for the total amount of cows on any given day (h) and $C P D=$ cost per hour of technical service $(\$ / \mathrm{h})$. The cost of pregnancy diagnosis by blood testing could be determined simply by the market cost of this service. Similar to the other 2 options, it would be calculated on a per cow per service basis.

Pregnancy diagnosis for programs A and $\mathrm{C}$ was performed weekly at $39 \mathrm{~d}$ after a previous TAI by transrectal palpation at a per-hour cost for veterinary palpation services of $\$ 105$. On the other hand, first pregnancy diagnosis for program B was performed on d 29 postTAI by transrectal ultrasonography at a per-hour cost of $\$ 135$.

Cost of Reproductive Services Associated With AI After ED. The cost of ED was calculated for all programs by multiplying the number of personnel involved in daily ED by the number of hours per day performing the task by the cost per hour of labor. This calculation was adjusted to effective days of ED to account for the fact that not all lactating cows are available for ED in a given year when the calving interval is greater than 12 mo as observed in the case study: $E D C_{s}$ $=(P E R \times H \times C E D)_{s} /\left(L C O W S_{s} \times 12 / C I\right)$ [27], where $E D C=\mathrm{ED}$ cost per service $(\$ /$ service $), P E R=$ number of personnel involved in ED (no.), $H=$ number of hours per day performing ED (no.), $C E D=$ cost per hour of labor to perform $\mathrm{ED}(\$ / \mathrm{h}), L C O W S=$ lactating cows (no.), and $C I=$ current calving interval for the herd (mo).

Estrous detection cost was used to calculate cost of a service for the reproductive program that used $100 \%$ ED and in cases when the programs combined the use of synchronization protocols and ED for AI. For the $100 \%$ ED program, calculation of AI and pregnancy diagnosis cost was similar to that of cows bred after synchronization and resynchronization of ovulation. For the case study, ED cost was set at $\$ 15.00 / \mathrm{h}$ and was assumed to be performed by 1 farm employee $7 \mathrm{~d} /$ wk for a total of $4 \mathrm{~h} / \mathrm{d}$. The calving interval was set at $14.1 \mathrm{mo}$.

Milk Production. The variable milk production was defined as the total expected milk production of 
a cow dependent on the time of pregnancy or culling because of reproductive failure. For instance, a cow becoming pregnant at 80 DIM $(\mathrm{DO}=80)$ will end her lactation around $360 \mathrm{~d}$ postpartum. If the cow is dried off $60 \mathrm{~d}$ before parturition, the cow would have an effective $300 \mathrm{~d}$ of milk production (DIM $=1$ to 300 ). Conversely, if a cow does not become pregnant by 250 DIM and the cow is culled, the total milk production is that accumulated during those $250 \mathrm{~d}$ (DIM $=1$ to 250 ). The daily milk production for a cow on a particular farm will greatly depend on many factors related to each cow's characteristics and on the historical farm production. Therefore, lactation information from cows for the specific farm being analyzed was entered in the model to generate lactation curves. Total number of cows and lactation performance for parity groups 1, 2, and $>2$ were entered separately to allow an economic evaluation for the specific parity groups (primiparous or multiparous) or for the entire herd. Alternatively, if a herd for analysis lacks lactation records, reference lactation curves for primiparous and multiparous cows for defined rolling herd average fluctuating between 8,170 and $13,620 \mathrm{~kg} /$ cow per year from 3.6 million Wisconsin records (data provided by AgSource Cooperative Services, Verona, WI) are embedded in the model and may be used.

Lactation curves for the case study pertaining to the specific farm were obtained from a DairyComp305 file. Data were retrieved from the file corresponding to November 2008 when the synchronization of ovulation study used to retrieve reproductive performance data concluded (Giordano et al., 2009). Average milk production at first test for first-parity cows was $28 \mathrm{~kg} / \mathrm{cow}$ per day, whereas for multiparous cows it was $40 \mathrm{~kg} /$ cow per day with a rolling herd average of $13,354 \mathrm{~kg} /$ cow. Peak milk production occurred at the fourth test with $43 \mathrm{~kg} /$ cow per day for primiparous cows and at the third test with $51 \mathrm{~kg} /$ cow per day for multiparous cows. Milk production information was available for 14 tests or 405 DIM and future milk production beyond this time point was projected by continuing the milk production decay observed between the peak and last test milk production.

Feed DM Intake For Lactating Cows. Feed DM intake was calculated as $2 \%$ BW plus $30 \%$ of FCM as defined in equation [28] (VandeHaar et al., 1992). This was consequently calculated based upon the lactation curve according to parity and production level. Milk tests of the case study farm indicated the milk to contain, on average, $3.5 \%$ fat: $F I_{D I M}=[(B W \times 0.02)+$ $\left.0.3 \times\left(4 \% F C M_{D I M}\right)\right],[28]$, where $B W=$ body weight $(\mathrm{kg} / \mathrm{cow}), F C M=$ fat-corrected milk calculated as $4 \%$ $\mathrm{FCM}(\mathrm{NRC}, 2001)$, and $F C M_{D I M}=4 \% \times M P_{D I M}+15$ $\times F A T$ [29], where $M P=$ milk production and $F A T=$ milk fat (\%).

Involuntary Culling Risk. The involuntary culling risk (remaining culling after subtracting mortality risk and culling because of reproductive failure) by parity was compiled monthly from De Vries (2004) and converted to daily culling risk from DIM $=1$ to 720 . Once the general curve of culling was defined, it was adjusted to match the farm predefined culling risk. Farm involuntary culling risk for the case study was obtained from the DairyComp305 file and was 29.4\%/yr. Involuntary culling was obtained by calculating the proportion of cows that left the herd during a 1-yr period for any reason except death or reproductive failure.

Unexpected Death Risk. The death risk was defined as a proportion of the involuntary culling risk as found on the Wisconsin's Benchmarks (AgSource Cooperative Services, Verona, WI).The unexpected death risk for the case study was obtained from the farm's DairyComp305 file and was $6.9 \% / y r$.

Definition of Economic Variables. To perform multiple cost and revenue calculations, the model required specific economic parameters, including price of milk, salvage value of cull cows, market value of newborn calves, feeding cost for lactating and dry cows, and the discount rate of money. Table 3 presents the specific values used by the model for the case study. The values shown were obtained from the Wisconsin Calculated Milk Cost of Production report for November 2010 published by the Center of Dairy Profitability at the University of Wisconsin-Madison (http://cdp. wisc.edu/).

\section{Sensitivity Analysis}

Sensitivity analyses were used to determine the effect of changes in specific inputs on the outcomes while maintaining the values of all other parameters constant. After the initial simulations, the studied programs were

Table 3. Economic variables (November 2010 ) used in the case study to compare 3 different reproductive management programs in a commercial dairy herd in Wisconsin

\begin{tabular}{lc}
\hline Economic variable & Value \\
\hline Milk price $(\$ / \mathrm{kg})$ & 0.37 \\
Salvage value of a cow $(\$ /$ cow $)$ & 624 \\
Market value of a female calf $(\$ /$ calf $)$ & 108 \\
Market value of a male calf $(\$ /$ calf $)$ & 44 \\
Value of a heifer replacement $(\$ /$ heifer $)$ & 1,288 \\
Feed cost for lactating cows $(\$ / \mathrm{kg}$ of $\mathrm{DM})$ & 0.22 \\
Feed cost for dry cows $(\$ /$ cow per day) & 2.20 \\
Discount rate $(\% / \mathrm{yr})$ & 6.5 \\
\hline
\end{tabular}

${ }^{1}$ Center for Dairy Profitability at the University of Wisconsin-Madison (http://cdp.wisc.edu/). 
ranked based on their NPV. Subsequently, sensitivity analyses were performed to determine the level of CR required for the economically inferior $100 \%$ TAI program to generate a similar or greater NPV than that of the superior TAI program. The CR was increased sequentially by 1 percentage point within a 15 -percentagepoint range to determine the $\mathrm{CR}$ at which the NPV for the inferior program surpassed the value of the superior one. Similarly, for comparing the $100 \%$ ED program and the TAI programs, sensitivity analyses were used to determine the level of increased or decreased in SR for the $100 \%$ ED required to generate similar values of NPV as the $100 \%$ TAI programs. In addition, sensitivity analyses were carried out to explore the effect of adding ED between TAI for second and subsequent AI services in programs A and B. The proportion of cows inseminated after ED was sequentially increased by 10 percentage points from 10 to $80 \%$ for both of the $100 \%$ TAI programs. The CR for AI after estrus was assumed similar to that of program C (30\%) and remained constant throughout all AI services.

\section{RESULTS AND DISCUSSION}

\section{Decision Support System Overview}

The first objective of this study was to present a decision support system developed from a mathematical simulation model intended to forecast differences in NPV associated with the on-farm application of diverse reproductive management strategies in dairy herds (available at http://dairymgt.info/tools.php $\rightarrow$ Reproduction $\rightarrow$ UW-DairyRepro\$: A Reproductive Economic Analysis Tool). An interesting attribute of this model is that it is highly versatile and adaptable to the conditions on each dairy farm and thus is capable of performing customized economic evaluations. To accommodate the multiple reproductive management practices used on modern dairy farms, the model is capable of comparing programs that rely solely on ED, synchronization of ovulation and TAI, or combinations thereof. A thorough description of the reproductive parameters is essential to generate detailed calculations of all the costs and revenues associated with reproductive management and performance. In this regard, numerous ovulation synchronization protocols commonly used in the dairy industry were predefined and loaded into the support system to be selected at the time of defining reproductive programs to be compared. Once the programs are selected, a thorough description of the resources used to perform the programs follows to generate a complete calculation of AI breeding costs, including hormones for synchronization, labor cost for injections, AI, and pregnancy diagnosis. Additionally, several productive and economic parameters are needed throughout the different modules to account for the various interactions between factors affecting economic results. The ultimate goal of this support system is to assist dairy producers in the process of selecting the best reproductive management strategy. The direction and magnitude of NPV differences between the programs compared permits identifying the best reproductive management program to perform at the farm under study.

\section{Economic and Reproductive Outcomes for Case Study}

Under the conditions of the case study simulated for a high-producing commercial herd in Wisconsin, the model indicated that the best reproductive program to apply on this specific farm was program B (Table 4), which used $100 \%$ TAI after synchronization of ovulation with Double-Ovsynch for all AI breedings. Program B presented the greatest NPV of $\$ 2,360 \mathrm{cow} / \mathrm{yr}$ compared with $\$ 2,336 \mathrm{cow} / \mathrm{yr}$ for program A (100\% TAI with Double-Ovsynch and D32 Resynch) and $\$ 2,291$ cow/yr for program C (100\% ED). When compared with the baseline 100\% ED program (C), these differences represented an advantage of $\$ 45$ and $\$ 69$ cow/yr for programs $\mathrm{A}$ and $\mathrm{B}$, respectively. Moreover, the difference between the two $100 \%$ TAI programs was $\$ 24$ cow/yr in favor of program B. Overall, the greater benefit of performing either one of the 100\% TAI instead of the $100 \%$ ED program is not surprising because the differences in reproductive performance simulated by the model were significant (Figure 1). Conversely, the better economic performance of program B over program A was surprising given the more labor-intensive resynchronization of ovulation protocol that requires double the number of hormone injections and the extended IBI. Despite having a 7-d-longer IBI, program B outperformed program A, which had lower CR (9\%) for Resynch AI breedings. Taken together, these results suggest that the improvement expected by having in place a simpler and less expensive protocol with shorter IBI, as in program A, was counterbalanced by the better fertility achieved by Resynch AI breedings after applying the DoubleOvsynch Resynch protocol in program B. Results from this case study are highly dependent on the reproductive performance data. Hence, other results may be observed under different scenarios.

The simulated herd reproductive dynamics generated by the model showed a clear advantage for both $100 \%$ TAI programs over the 100\% ED program and slightly better performance for program B over program A (Figure 1, Table 5). Despite having an extended duration of VWP (32 d) and fewer AI attempts (6 for 100\% 


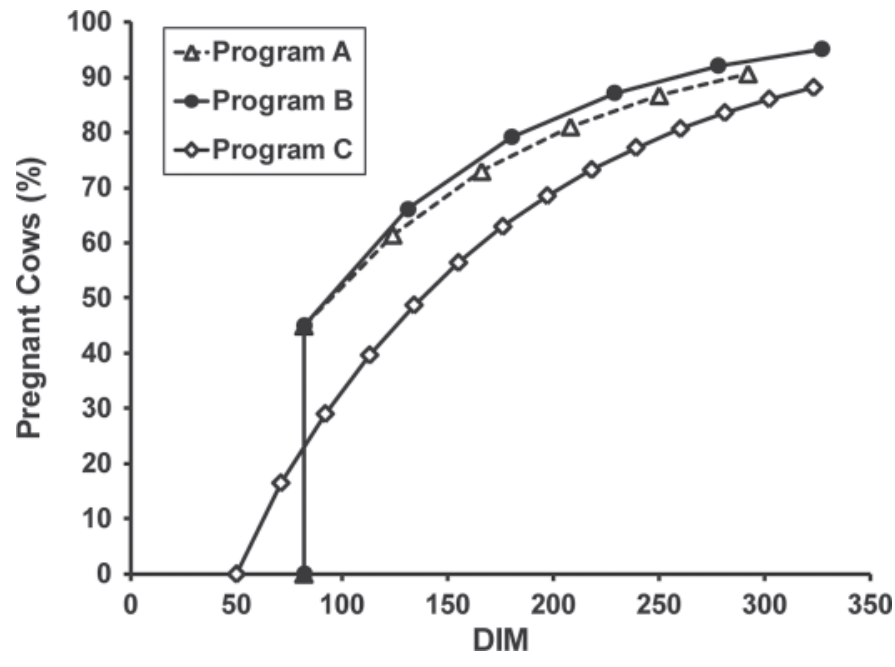

Figure 1. Survival curves for pregnancy for the 3 reproductive management programs compared in the case study based on simulated herd dynamics with input parameters. Synchronization of ovulation was used for all AI breedings in programs A (Double-Ovsynch and D32 Resynch) and B (Double-Ovsynch and Double-Ovsynch Resynch), whereas AI was performed after estrous detection (ED) in program C. The voluntary waiting period (VWP) for programs A and B was $82 \mathrm{~d}$, whereas it was $50 \mathrm{~d}$ for program C. After the end of the VWP, cows were inseminated until 330 DIM.

TAI vs. 13 for $100 \%$ ED) until the predefined cut-off of 330 DIM, both 100\% TAI programs outperformed the $100 \%$ ED program. This difference was predictable because one of the major advantages of TAI programs for first service is the high SR obtained in the first cycle after the end of the VWP because $100 \%$ of the cows are inseminated at a fixed time within a short window.
In addition to the increased SR for first service, the increased CR obtained with Double-Ovsynch resulted in $45 \%$ of the total available cows becoming pregnant by 82 DIM. After first-service AI, the combination of a consistently high AI submission rate attained with the resynchronization protocols and acceptable CR (30\% for program A and 39\% for program B) maintained the differences in performance. In contrast, with a VWP of $50 \mathrm{~d}$ and an SR of $50 \%$, the $100 \%$ ED program did not compensate for the lower $\mathrm{CR}$ at first service $(33 \%)$ because this management scheme required at least 4 complete 21 -d breeding cycles occurring within approximately 130 DIM to achieve the same number of pregnant cows as the TAI programs at 82 DIM. After first service, the differences remained probably due to the higher likelihood for the cows on the TAI programs to be inseminated and become pregnant in spite of the longer IBI that resulted in only 6 total AI services compared with the 13 attempts on the $100 \%$ ED program. On the other hand, the major difference between programs $\mathrm{A}$ and $\mathrm{B}$ was their resynchronization strategies because they purposely had similar synchronization protocols and results for first-service postpartum TAI. Regardless of the longer IBI for program B, the 330 cut-off DIM value allowed for a total of $6 \mathrm{AI}$ attempts in both programs, but the higher CR obtained by program $\mathrm{B}$ resulted in a greater percentage of pregnant cows (95.4 vs. $90.8 \%$ for program B and A, respectively) by the end of the breeding period.

Based on the simulated herd dynamics calculated from the existing or predicted input reproductive performance data (i.e., VWP, IBI, SR, and CR to AI services),

Table 4. Calculated economic values for the components of the net present value (NPV) for the 3 different reproductive management programs compared in the case study

\begin{tabular}{|c|c|c|c|}
\hline \multirow[b]{2}{*}{ Component $^{1}$} & \multicolumn{3}{|c|}{ Program $^{2}$} \\
\hline & $\mathrm{A}$ & $\mathrm{B}$ & $\mathrm{C}$ \\
\hline Milk income over feed cost & 2,623 & 2,636 & 2,561 \\
\hline Milk value & 4,235 & 4,266 & 4,142 \\
\hline Feed cost milking period & 1,488 & 1,502 & 1,464 \\
\hline Feed cost dry period & 125 & 129 & 117 \\
\hline Total culling cost & -288 & -274 & -284 \\
\hline Involuntary culling pregnant $^{3}$ & -242 & -252 & -234 \\
\hline Involuntary culling nonpregnant ${ }^{3}$ & -14 & -7 & -15 \\
\hline Reproductive culling & -32 & -16 & -34 \\
\hline Reproductive program cost & -54 & -58 & -37 \\
\hline Income from newborn & 55 & 56 & 51 \\
\hline Value of reproductive program (NPV) & 2,336 & 2,360 & 2,291 \\
\hline Value over $100 \%$ ED program & 45 & 69 & - \\
\hline
\end{tabular}


Table 5. Reproductive outcomes for the 3 different reproductive management programs compared in the case study

\begin{tabular}{lccc}
\hline & \multicolumn{3}{c}{ Program } \\
\cline { 2 - 4 } Reproductive parameter & $\mathrm{A}$ & $\mathrm{B}$ & $\mathrm{C}$ \\
\hline 21-d pregnancy rate (\%) & 22 & 25 & 15 \\
21-d service rate (\%) & 62 & 60 & 50 \\
Average conception rate for all AI services (\%) & 38 & 42 & 32 \\
Average days open (d) & 131 & 136 & 151 \\
Projected calving interval (mo) & 14.1 & 14.9 \\
'Synchronization of ovulation was used for all AI breedings in programs A (Double-Ovsynch and D32 Resynch \\
at first and second or subsequent service, respectively) and B (Double-Ovsynch and Double-Ovsynch Resynch \\
at first and second or subsequent service, respectively), whereas AI was performed after estrous detection (ED) \\
in program C. The voluntary waiting period (VWP) for programs A and B was 82 d, whereas it was 50 d for \\
program C. After the end of the VWP, cows were inseminated until 330 DIM.
\end{tabular}

the model calculated a set of parameters widely used to monitor reproductive performance of dairy herds (i.e., 21-d PR, 21-d SR, average CR, DO, and PCI; Table 5). Similar to the results for NPV, the values obtained for all the simulated reproductive parameters were only an approximation; hence, they should be regarded only within the context of comparing the programs for the case study. The greatest 21 -d PR was $25 \%$ for program B, followed by $22 \%$ for program A, and finally $15 \%$ for program C. These results are in agreement with the survival curve for pregnancy generated by the model (Figure 1) that showed the different rates at which cows became pregnant after the end of the VWP. As previously discussed, the main contributors to the better performance of the $100 \%$ TAI programs were the higher SR and CR at first service, which resulted in large differences in the proportion of pregnant cows before 100 DIM. Afterward, the consistently high SR and good overall CR for the TAI programs maintained that difference until the end of the breeding period at 330 DIM. The greater 21-d PR for program B over A suggests that the better $\mathrm{CR}$ for second and subsequent AI services in program $\mathrm{B}$ outweighed the shorter IBI of program A (42 d and $49 \mathrm{~d}$ for A and B, respectively) that resulted in a $2 \%$ greater 21 -d SR (Table 5).

Average values for $\mathrm{CR}$ for all AI services followed the same order as 21-d PR, which is not surprising given they only represent a weighted average of the values entered as inputs. The differences in DO between the 100\% ED and TAI programs were expected and clearly reflected the difference in the rate at which the cows became pregnant for these 2 different types of programs. Conversely, when comparing the two $100 \%$ TAI programs, the results seemed in conflict at first because DO were greater for program $\mathrm{B}$ than for $\mathrm{A}$. Because average DO is simply an indication of the DIM at which cows conceived, the greater DO for program $\mathrm{B}$ reflected the 7-d-longer IBI for resynchronized breedings. In spite of having a timing disadvantage for AI services, creating a greater proportion of pregnant cows after each service resulted in better economic results for program B than for A. Finally, PCI was also a reflection of better performance of the $100 \%$ TAI versus the $100 \%$ ED program, whereas the better PCI for program $\mathrm{B}$ over A was primarily due to the different proportion of pregnant cows at the end of the breeding period. In the PCI calculation, nonpregnant cows at the end of the breeding period were assumed to conceive on that date and then the next calving is projected $280 \mathrm{~d}$ afterward; thus, the program with the greatest proportion of nonpregnant cows (program A) had the greater PCI.

The economic components of the NPV for each one of the compared programs in the case study were broken down to assess the contribution that each one had in the total value of the reproductive program (Table 4). Consistently throughout the programs, the major contributor to NPV was income over feed cost, which is the difference between the total milk value (i.e., milk sales) and the cost of feeding cows during their lactation and dry period (i.e., feeding cost). The greatest values were for program B, followed by $\mathrm{A}$ and $\mathrm{C}$. It was not surprising to observe that, in proportion, milk value was the major factor adding to the NPV because the single major source of income for dairy farms is milk sales (White et al., 2002; De Vries, 2004). In addition, feeding cost during the lactation period was the greatest cost among the factors considered for analysis. This is also in agreement with the reality of dairy operations because one of the major costs of production is feeding cost (White et al., 2002; De Vries, 2004). The greatest milk value for the programs with better reproductive performance (B and $\mathrm{A}$ ) indicated that having a greater proportion of pregnant cows at an appropriate time during lactation improved the revenue as opposed to having fewer cows pregnant and later in their lactations when DEMV declined significantly.

Total culling costs were the highest in programs A and $\mathrm{C}$, whereas culling cost was significantly lower for 
B (Table 4). Involuntary culling (that included mortality cost) of pregnant cows represented between 82 and $92 \%$ of the total cost of culling; however, in proportion, the differences between programs were smaller than the differences for involuntary culling of nonpregnant (includes mortality cost) and culling due to reproductive failure. Culling of pregnant cows was the greatest contributor to total culling cost because, in all 3 programs, between 88.1 and $95.4 \%$ of the cows became pregnant so the rate of culling applied to a larger population of cows when compared with nonpregnant cows after the last service. On the other hand, the major contributors to the higher total culling cost for programs $\mathrm{A}$ and $\mathrm{C}$, when compared with program $\mathrm{B}$, were the cost of involuntary and voluntary culling for nonpregnant cows after the cut-off DIM for AI services, which in both cases was more than double that of program B (Table 4). This difference was largely explained by the different proportion of nonpregnant cows at the 330 DIM cut-off in each program, which was the highest for program C (11.9\%) followed by A $(9.2 \%)$, and the lowest for B (4.6\%). The lower proportion of nonpregnant cows in program B was clearly a consequence of the better reproductive performance during the breeding period. As a result, this program had more pregnant cows and fewer reproductive culls, which are always costly because the value of a culled cow is lower than that of a heifer ready to calve and start a new lactation even when the added value of the newborn calf coming with the heifer is included as stipulated by the model.

Among all factors considered for the calculation of the value of the reproductive programs, the overall breeding costs had the lowest contribution to the final NPV (Table 4). This finding suggests that the impact of reproductive management program costs in the expenses for the dairy could be minimal compared with the advantage of the improved reproductive efficiency, because the most expensive program (B) still had the greatest NPV. When assessing the total reproductive program cost, we were not surprised to observe that the highest value was for program B followed by A, and finally C. The total calculated cost of an AI (i.e., total AI service cost included hormones, labor, AI, and pregnancy diagnosis) for first-service postpartum was $\$ 29.20$ for programs A and B and $\$ 13.10$ for program $\mathrm{C}$, whereas the cost for second and subsequent AI service postpartum was $\$ 21.00, \$ 29.20$, and $\$ 13.10$ for programs A, B, and $\mathrm{C}$, respectively. Additionally, for cows in programs $\mathrm{A}$ and $\mathrm{B}$ after first-service postpartum TAI, cows becoming pregnant after each breeding attempt received the extra cost of being re-enrolled in the resynchronization protocol (hormones plus labor for injections) for the next TAI that was not completed after being diagnosed pregnant at $39 \mathrm{~d}$ for program $\mathrm{A}$ and $29 \mathrm{~d}$ for program B. The added cost was $\$ 3.50$ for program A and $\$ 4.70$ for program B. The difference between the $100 \%$ TAI programs and the $100 \%$ ED program was due to the ovulation synchronization protocols that require the administration of hormone injections adding extra cost for hormones and the labor to administer injections. Implicit to the programs used in the case study, the cost of first-service AI was equal for programs A and B because both used Double-Ovsynch for first-service TAI, whereas they differed on the cost of second and subsequent AI because program A used D32 Resynch and program B used Double-Ovsynch Resynch. Even after careful consideration of all the factors that added cost to the programs, the use of the more complex and expensive one, which had the greatest reproductive cost, resulted in the greatest NPV. Taken together, these observations suggest that at the time of selecting a reproductive management strategy for a herd, it is important to consider not only the added cost of applying the program, but also the remarkable effect that it may have in the overall reproductive performance and hence the profitability of the operation.

In contrast to reproductive program cost, income from newborn calves was the greatest for program B, intermediate for $\mathrm{A}$, and lowest for $\mathrm{C}$, also reflecting the better reproductive performance for the $100 \%$ TAI programs because income from newborn calves was directly related to the number of pregnancies generated. The differences in this parameter can be entirely attributed to reproductive efficiency because, in the calculation of culling cost, the model already accounted for the added value of newborn calves from heifers replacing culled cows due to reproductive failure.

\section{Expected Monetary Value}

An intermediate calculation of the model was the EMV for pregnant and nonpregnant cows. The variation in EMV by DIM for pregnant and nonpregnant cows for the case study is shown in Figure 2. This value indicates the net revenue $(\$ /$ cow per day) generated by cows throughout their lactation when they become pregnant at specific DIM (or by the last DIM before being culled for nonpregnant cows). The DIM at which the EMV reaches maximum value may be used as an indicator of the most appropriate stage of the lactation for cows to become pregnant. Conversely, the EMV for nonpregnant cows, which is always lower than that of pregnant cows, is an indication of the profit generated by those cows before being culled and replaced with a heifer. It is interesting that the EMV for pregnant cows increased from a value of $\$ 6.96$ at 50 DIM to a maximum of $\$ 7.07$ at 130 DIM, showing very small variation from 90 to $130 \mathrm{DIM}$ and then declining more 
steeply after 150 DIM to reach the lowest value of $\$ 6.13$ by 330 DIM, when cows were no longer inseminated. If considered on a yearly basis $(\$ /$ cow per year), the difference between a cow becoming pregnant immediately after the VWP at 50 and 130 DIM (when EMV reached its maximum) was $-\$ 40$, whereas the difference when comparing 130 to 330 DIM (when EMV reached the lowest value) was $-\$ 343$. These data support the notion that an optimal time exists for lactating dairy cows to become pregnant in their lactation and that conceiving too early or too late is less profitable for the dairy operation. In particular, for the specific dairy farm studied, and under the conditions analyzed in the case study, the optimal timing seemed to be around 130 DIM. Moreover, the dramatic change in EMV observed after the peak suggests that the greatest losses occurred when cows became pregnant beyond 150 DIM. The difference in EMV for pregnant cows between 2 specific DIM could also be interpreted as the cost of days open. Numerous studies using multiple techniques and models estimated the cost of extended lactations resulting from additional days open in lactating dairy cows (Holmann et al., 1984; Plaizier et al., 1997; Meadows et al., 2005). Results vary widely due to the different methodologies and input data used for calculation. In agreement with our results, most studies reported a nonuniform cost for days open, with lower costs immediately before and after the optimal time for conception followed by significant increases in costs for days open as DIM progressed (Holmann et al., 1984; Plaizier et al., 1997; Meadows et al., 2005). After taking into account the resulting herd dynamics from the 3 programs compared, the cost associated with their application, and the EMV for pregnant and nonpregnant animals, the model calculated the greatest NPV for the 100\% TAI programs, which generated the greatest number of pregnancies by the end of the breeding period. It seems plausible that the major economic advantage for these 2 programs when compared with the $100 \%$ ED was that more than $60 \%$ of the total eligible cows became pregnant between 82 and 150 DIM (Figure 1) when the EMV reached its maximum values and before it started to decline significantly (Figure 2).

\section{Sensitivity Analyses for Case Study}

The first sensitivity analysis for the case study was performed to demonstrate the flexibility of the model to accommodate multiple scenarios and give some interesting insights into the economic behavior of the different reproductive programs compared. As previously discussed, under the conditions of the initial simulation, program B (with a more expensive resynchronization of ovulation protocol and longer IBI) was superior to program A by $\$ 24 /$ cow per year. When 1-percentagepoint increments were added to the $\mathrm{CR}$ for second and subsequent TAI in program A (while other input parameters remained constant), a CR of $34 \%$ generated a NPV slightly greater than that of program B $(\$ 2,364$ vs. $\$ 2,360 /$ cow per year for programs A and B, respectively); with a CR of 35\%, program A surpassed the NPV of program B by $\$ 10 /$ cow per year (Figure 3 ). Interestingly, these results show that relatively small changes in CR have a significant effect on the economic outcomes because a 1-percentage-point increment in $\mathrm{CR}$ within the range of $\mathrm{CR}$ tested in the sensitivity analysis represented $\$ 8$ to $\$ 4 /$ cow per year of extra income for the farm. The CR required for program A to generate similar NPV to program B is achievable by dairy farms with good reproductive management and has been reported in some studies using the Ovsynch protocol for resynchronization of ovulation and TAI in lactating dairy cows (Sterry et al., 2006; Thompson et al., 2010). When the CR for second and subsequent TAI of program A was increased to the same level as in program B $(39 \%)$, the NPV was $\$ 32 /$ cow per year higher for program A over program B. Although this is a very unlikely scenario for resynchronized TAI after using the Ovsynch protocol in lactating dairy cows, it

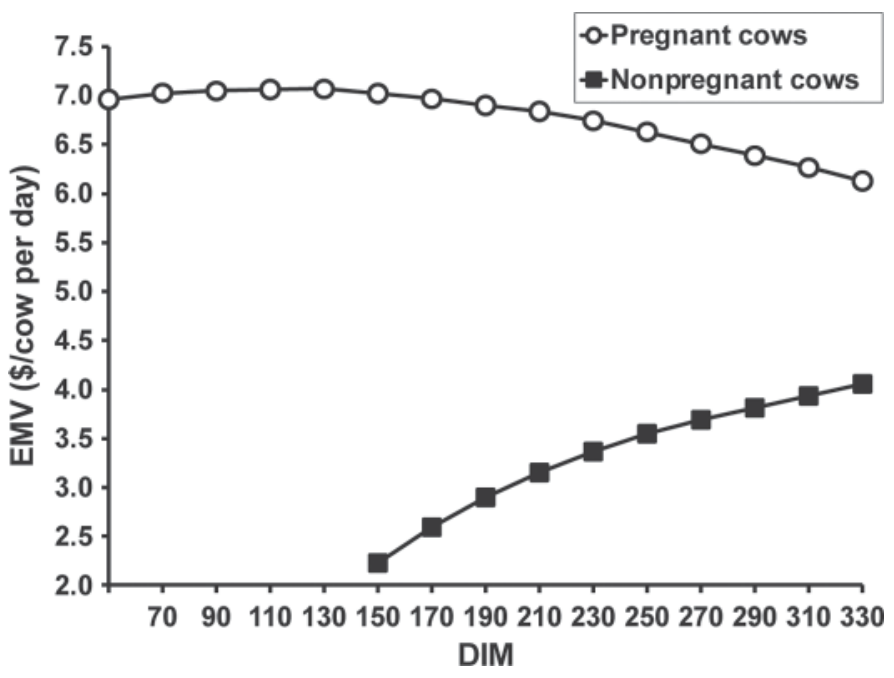

Figure 2. Variation in expected monetary value (EMV; $\$ /$ cow per day) by DIM for pregnant and nonpregnant cows calculated by the model based on productive, reproductive, and economic parameters entered as inputs for the case study. Values reported for pregnant cows span from 50 to 330 DIM, representing the period from the end of the voluntary waiting period to cut-off DIM for breeding in the program with the longest breeding period (program C). For nonpregnant cows, the represented period spans from 150 to 330 DIM to illustrate the increase in EMV by DIM. As opposed to pregnant cows that will be assigned an EMV individually based on the DIM at pregnancy, for nonpregnant cows only one value of EMV applies to all cows culled due to reproductive failure at the end of the breeding period (i.e., $\$ 6.13$ at DIM for the case study). 
illustrates the major benefit of having in place a less expensive protocol with a 7-d-shorter IBI. On the other hand, the sensitivity analysis showed that for program $\mathrm{B}, 1$-percentage-point increments in $\mathrm{CR}$ increased the NPV of the program by $\$ 9$ to $\$ 4 /$ cow year with the greatest benefits at low levels of CR.

Because of the small contribution that total AI cost made to the total reproductive program value, it seems reasonable to suggest that when the $\mathrm{CR}$ for second and subsequent TAI was the same, the difference in NPV between programs was essentially due to the shortened IBI for program A. Likewise, these data may be interpreted and used in practical terms to determine the minimum level of $\mathrm{CR}$ required for second and subsequent TAI in program B to be profitable for the dairy farm. Interestingly, these observations suggest that the economic success of programs with extended IBI is highly dependent on obtaining high CR for second and subsequent AI services, whereas those programs with shorter IBI may be more profitable regardless of a lower CR. In contrast, sensitivity analyses for the comparison between programs $\mathrm{B}$ and $\mathrm{C}$ compared 1-percentagepoint increments in SR for the 100\% ED (program C) rather than changes in CR because, under most circumstances for dairy herds performing AI after detection of estrus, SR is more easily improved than CR.

To generate an NPV similar to that for program B, the $100 \%$ ED program $(\mathrm{C})$ required a 12 -percentagepoint improvement in SR from 50 to $62 \%$ (maintaining the $\mathrm{CR}$ ), whereas an additional 1-percentage-point increase in SR beyond $62 \%$ was sufficient to outperform program B $(\$ 2,364$ vs. $\$ 2,360 /$ cow per year for programs C and B, respectively; Figure 3). Unfortunately, during the course of the study used to provide the data for the $100 \%$ TAI programs, ED was not performed at this dairy farm; therefore, it is uncertain if a $62 \%$ SR was achievable at this particular farm. Although this level of SR is observed on some dairy farms applying very efficient ED programs, epidemiological data indicated, for example, that only a very small proportion of dairy herds in the United States (Schefers et al., 2010) and Canada (Campbell, 2009) had 21-d SR $\geq 60 \%$.

Other sensitivity analyses were performed to determine if adding ED between TAI for second and subsequent AI services would have a beneficial or detrimental effect on the economic outcomes for the case study. Interestingly, the changes in NPV for programs $\mathrm{A}$ and $\mathrm{B}$ followed opposite trends when increasing the proportion of cows AI after estrus (Figure 4). Whereas NPV showed a positive trend at all levels of ED for program A, that for program B decreased at all levels of ED. The different behavior observed for both programs reflected the differences in IBI and CR. Adding

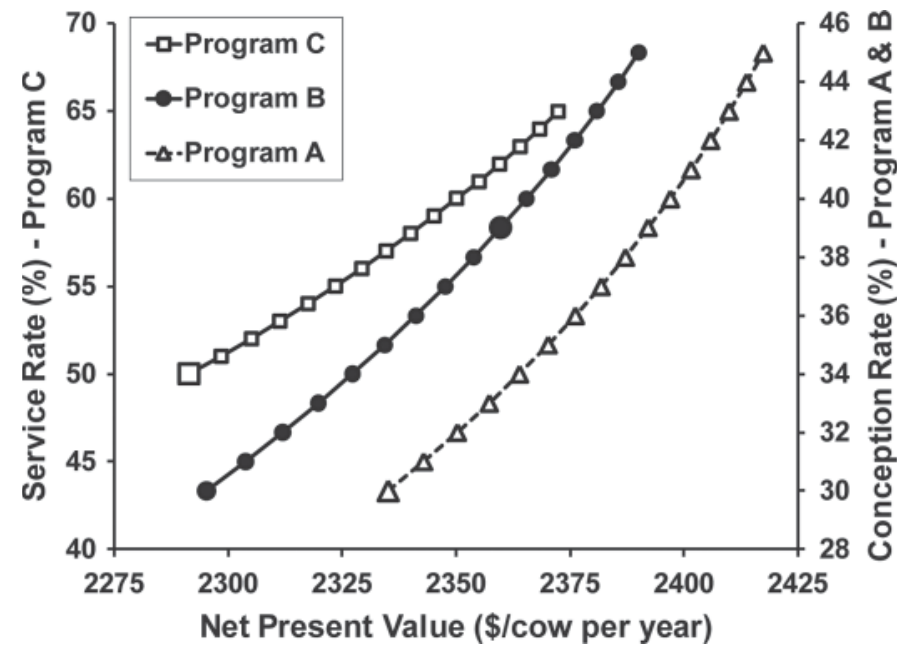

Figure 3. Variation in net present value (NPV; $\$ /$ cow per year) after sensitivity analyses. Synchronization of ovulation was used for all AI breedings in programs A (Double-Ovsynch and D32 Resynch) and B (Double-Ovsynch and Double-Ovsynch Resynch), whereas AI was performed after estrous detection (ED) in program C. For programs A and $\mathrm{B}$, conception rate $(\mathrm{CR})$ for second and subsequent TAI services varied by 1 percentage unit in a range of 30 to $45 \%$, while first $\mathrm{AI}$ service CR remained constant. For program C, service rate (SR) varied by 1 percentage unit from 50 to $65 \%$ for all AI services, while CR remained constant. The enlarged marker in each line represents SR (program C) or CR (programs A and B) entered in the initial simulation.

ED was advantageous for program A because the CR of AI services after estrus was similar to that of the TAI. Therefore, cows that received AI at estrus $21 \mathrm{~d}$ after the previous TAI service had a $50 \%$ reduction in their IBI that otherwise would be $42 \mathrm{~d}$ if they were bred according to protocol. The opposite was observed for program B because the CR to the TAI was significantly greater than that of the AI services after ED (39 vs. $30 \%$, respectively).

Despite having a shorter IBI (21 vs. 49 d), cows receiving AI at estrus had a lower chance of becoming pregnant than if inseminated after completing the resynchronization protocol with program B. Because the model stipulates that cows having AI at estrus failing to conceive are not eligible for breeding to the following TAI, they can only receive their next AI after detection of estrus in the 21-d period following the TAI or be enrolled in the resynchronization protocol to receive the following TAI. As a result, these cows will have a prolonged IBI beyond the $49 \mathrm{~d}$ if they were bred on TAI after Double-Ovsynch Resynch in program B and resulted nonpregnant. Based on these results, the combination of ED with a TAI program is profitable when the CR of AI services at estrus are at least similar to that of TAI services but not when the CR are lower than that of TAI breedings even when the IBI after resynchronization is significantly greater. 


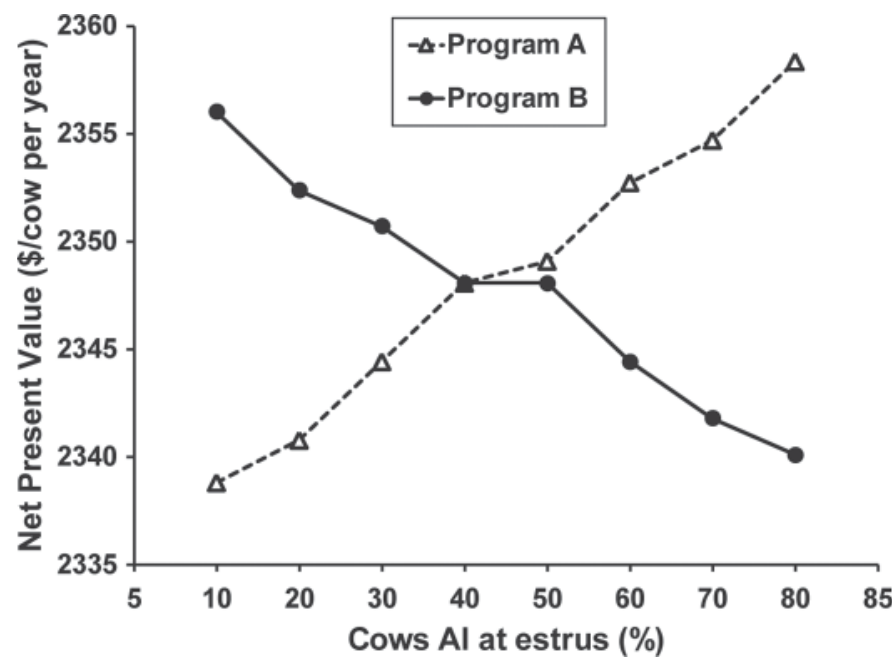

Figure 4. Net present value (NPV; \$/cow per year) for programs A and $\mathrm{B}$ when adding estrous detection (ED) in between timed AI (TAI) for second and subsequent AI services. Synchronization of ovulation was used for all AI breedings in programs A (Double-Ovsynch and D32 Resynch) and B (Double-Ovsynch and Double-Ovsynch Resynch). Sensitivity analysis was performed to determine the effect of adding ED for AI breedings in between resynchronized TAI. The proportion of cows inseminated after ED was sequentially increased by increments of 10 percentage units from 10 to $80 \%$ for both of the $100 \%$ TAI programs. The conception rate (CR) for AI after estrus was assumed similar to that of program $\mathrm{C}(30 \%)$ and remained constant throughout all AI services.

\section{Decision Support System Limitations}

As with any simulation or modeling approach, some limitations of the methodology are recognized, which in this study are related, in part, to the need to create a user-friendly framework with minimal processing time packaged as a practical and realistic decision support system. One limitation of the model is that all calculations are performed independently for groups of cows in different lactations; later, these results are aggregated at the herd level. For every cow in the different parity groups (primiparous vs. multiparous) a new lactation is simulated and, if pregnant during the breeding period, a cow is considered to conclude the respective lactation $280 \mathrm{~d}$ after the date of conception. After completion of the dry period, cows are considered to calve and the value of the newborn is added to the profits generated during such lactation. On the other hand, cows failing to become pregnant are considered to finish their lactation at the cut-off DIM by the end of the breeding period, when they are replaced by a pregnant heifer. The total cost of culling is then calculated by summation of the market cost of a pregnant heifer, newborn calf coming from the heifer, and the salvage value of the culled cow. A concern with the assumption of independent lactations is that the events throughout the entire productive life of a cow could not be simulated.
However, the model calculations are comprehensive, including the performance of cows from all parity groups and standardizing all values to $\$ /$ cow per day. This might have a certain effect on the results because the timing of events during one lactation (i.e., DIM at conception) may affect the future performance of cows in their following lactation. A disadvantage of the method to calculate the cost of culling could be that the supply of heifers from the dairy farm under consideration is not taken into account. Therefore, in some dairy farms with good reproductive performance and a sufficient supply of heifers, the actual heifer rearing cost may be lower than the market value of a pregnant heifer. Another disadvantage of this approach is that our model does not consider retention pay-off (Groenendaal et al., 2004; De Vries, 2006) to assess the economic value of cows leaving the herd. Hence, the culling cost does not account for potential future profitability differences between those cows leaving the herd and their replacements. Nonetheless, these assumptions apply equally to all reproductive programs evaluated and consequently its differential effect might be only minimal.

Another possible limitation of the model is that early pregnancy losses and abortions are not considered. Clearly, this would affect the herd dynamics and the economic outcome because a cost is associated with cows that fail to carry a pregnancy to term (Thurmond and Picanso, 1990; De Vries, 2006). Both of these assumptions, however, apply equally to all the reproductive management strategies simulated. Therefore, the overall effect on the final NPV for each program should be minimal. In those cases where the rate of pregnancy loss or abortion is a concern, this model limitation may be compensated by lowering the CR of AI services by a factor proportional to the rate of pregnancy loss between the first and second pregnancy diagnoses after AI. Another approach is to enter the CR value after the second pregnancy diagnosis (recheck), which, under most circumstances in commercial dairy operations, is performed when the rate of late embryonic or early fetal losses declined significantly (Santos et al., 2004). Finally, another assumption was that the duration of the estrous cycle was $21 \mathrm{~d}$ and therefore all AI services occurred by the end of each 21-d period after VWP concluded. This assumption conflicts with reality because nonsynchronized (i.e., no hormonal intervention) cows cycle independent of each other and show signs of estrus randomly. In fact, it has been reported that the mean duration of the estrous cycle in lactating dairy cows is $22.9 \pm 0.7$ (Sartori et al., 2004), not exactly $21 \mathrm{~d}$. The implication of this assumption for the model is that cows conceiving after AI at estrus during any day before the end of a 21-d period were computed as pregnant at later DIM than when they actually con- 
ceived. Economically, the major effect would be for the proportion of cows that showed estrus very early in the 21-d period as opposed to cows showing estrus close to the end of the period. On the contrary, this limitation might be counterbalanced by the potential benefit on the results for programs using ED because cows that actually showed estrus after $21 \mathrm{~d}$ were considered to become pregnant a few DIM earlier.

As previously discussed, an important attribute of this model is the flexibility to accommodate multiple productive, reproductive, and economic scenarios for the specific farm under consideration or even for hypothetical scenarios created to explore the benefits or losses associated with certain reproductive management changes in dairy herds. Varying the values for any particular input parameter or set of parameters might create a different scenario on its own. The sensitivity analyses performed for the case study are a clear example of the model's flexibility because they demonstrate the extra income expected from improving one specific reproductive program parameter and the minimum improvement required to warrant the use of a different reproductive program.

\section{CONCLUSIONS}

Because of the wide array of reproductive management strategies available for implementation in dairy herds, the ability to predict the future reproductive and economic performance of herds through decision support systems is a valuable tool to dairy producers and consultants. In this study, we presented a highly adaptable tool based on a mathematical model combining Markov chain simulation with partial budgeting to obtain the NPV of different reproductive management programs in dairy herds. To account for all factors related to reproductive management adding to the costs and generating revenue for the dairy operation, the model requires specific productive, reproductive, and economic input data to simulate the conditions resulting from the application of the different management strategies under consideration. Then, at the time of selecting the reproductive program with the greatest NPV, it is critical to focus on the value differences rather than on the absolute values because those are meaningful only within the context of the comparison. For the particular case study simulating the conditions of a commercial herd in Wisconsin, the model indicated that (1) the two $100 \%$ TAI programs were superior to the $100 \%$ ED program; (2) among the $100 \%$ TAI programs, program B outperformed A because its better CR counterbalanced the longer IBI; (3) despite being the most expensive, program $\mathrm{B}$ had the best economic performance; (4) following a reasonable increase in CR (4\%) for Resynch breedings, program A could outperform program B; (5) adding ED to the $100 \%$ TAI programs was only beneficial for the program with lower CR (program A); (6) the improvement in 21-d $\mathrm{SR}$ required for program $\mathrm{C}$ to have the same NPV as program B was $12 \%$; and (7) the ideal time for cows to become pregnant was around 130 DIM.

\section{ACKNOWLEDGMENTS}

This project was supported by Agriculture and Food Research Initiative Competitive Grant no. 2010-8512220612 from the USDA National Institute of Food and Agriculture and by Hatch project WIS01171 to P.M.F. The authors thank Jerry Guenther (Department of Dairy Science, University of Wisconsin-Madison, Madison) for his assistance with data collection and processing throughout this study.

\section{REFERENCES}

Arbel, R., Y. Bigun, E. Ezra, H. Sturman, and D. Hojman. 2001. The effect of extended calving intervals in high-yielding lactating cows on milk production and profitability. J. Dairy Sci. 84:600-608.

Britt, J. H. 1985. Enhanced reproduction and its economic implications. J. Dairy Sci. 68:1585-1592.

Britt, J. S., and J. Gaska. 1998. Comparison of two estrus synchronization programs in a large, confinement-housed dairy herd. J. Am. Vet. Med. Assoc. 212:210-212.

Campbell, M. S. 2009. The association of level of milk production with reproductive performance in Canadian dairy cattle. MS Thesis. University of Guelph, Guelph, ON, Canada.

Caraviello, D. Z., K. A. Weigel, P. M. Fricke, M. C. Wiltbank, M. J. Florent, N. B. Cook, K. V. Nordlund, N. R. Zwald, and C. L. Rawson. 2006. Survey of management practices on reproductive performance of dairy cattle on large US commercial farms. J. Dairy Sci. 89:4723-4735.

De Vries, A. 2004. Economics of delayed replacement when cow performance is seasonal. J. Dairy Sci. 87:2947-2958.

De Vries, A. 2006. Economic value of pregnancy in dairy cattle. J. Dairy Sci. 89:3876-3885.

Ferguson, J. D., and D. T. Galligan. 1999. Veterinary reproductive programs. Pages 131-137 in Proc. 32nd Annu. Mtg. Am. Assoc. Bovine Pract., Nashville, TN.

Folman, Y., M. Kaim, Z. Herz, and M. Rosenberg. 1984. Reproductive management of dairy cattle based on synchronization of estrous cycles. J. Dairy Sci. 67:153-160.

Giordano, J. O., M. C. Wiltbank, S. Bas, A. P. Cunha, R. A. Pawlisch, J. N. Guenther, and P. M. Fricke. 2009. Fertility after timed artificial insemination in lactating dairy cows resynchronized using Double-Ovsynch or standard Ovsynch. J. Dairy Sci. 92(E-Suppl. 1):188. (Abstr.)

Groenendaal, H., D. T. Galligan, and H. A. Mulder. 2004. An economic spreadsheet model to determine optimal breeding and replacement decisions for dairy cattle. J. Dairy Sci. 87:2146-2157.

Holmann, F. J., C. R. Shumway, R. W. Blake, R. B. Schwart, and E. M. Sudweeks. 1984. Economic value of days open for holstein cows of alternative milk yields with varying calving intervals. J. Dairy Sci. 67:636-643.

Lima, F. S., A. De Vries, C. A. Risco, J. E. Santos, and W. W. Thatcher. 2010. Economic comparison of natural service and timed artificial insemination breeding programs in dairy cattle. J. Dairy Sci. 93:4404-4413.

Losinger, W. C., and A. J. Heinrichs. 1996. Dairy operation management practices and herd milk production. J. Dairy Sci. 79:506514 . 
Louca, A., and J. E. Legates. 1968. Production losses in dairy cattle due to days open. J. Dairy Sci. 51:573-583.

Meadows, C., P. J. Rajala-Schultz, and G. S. Frazer. 2005. A spreadsheet-based model demonstrating the nonuniform economic effects of varying reproductive performance in Ohio dairy herds. J. Dairy Sci. 88:1244-1254.

Momcilovic, D., L. F. Archbald, A. Walters, T. Tran, D. Kelbert, C. Risco, and W. W. Thatcher. 1998. Reproductive performance of lactating dairy cows treated with gonadotropin-releasing hormone $(\mathrm{GnRH})$ and/or prostaglandin $\mathrm{F} 2 \mathrm{a}\left(\mathrm{PGF}_{2 \alpha}\right)$ for synchronization of estrus and ovulation. Theriogenology 50:1131-1139.

Moreira, F., C. Orlandi, C. A. Risco, R. Mattos, F. Lopes, and W. W. Thatcher. 2001. Effects of presynchronization and bovine somatotropin on pregnancy rates to a timed artificial insemination protocol in lactating dairy cows. J. Dairy Sci. 84:1646-1659.

NRC. 2001. Nutrient Requirements of Dairy Cattle. 7th ed. Natl. Acad. Sci., Washington, DC.

Oltenacu, P. A., T. R. Rounsaville, R. A. Milligan, and R. H. Foote 1981. Systems analysis for designing reproductive management programs to increase production and profit in dairy herds. J. Dairy Sci. 64:2096-2104.

Olynk, N. J., and C. A. Wolf. 2009. Stochastic economic analysis of dairy cattle artificial insemination reproductive management programs. J. Dairy Sci. 92:1290-1299.

Plaizier, J. C., G. J. King, J. C. Dekkers, and K. Lissemore. 1997. Estimation of economic values of indices for reproductive performance in dairy herds using computer simulation. J. Dairy Sci. 80:2775-2783

Pursley, J. R., M. O. Mee, and M. C. Wiltbank. 1995. Synchronization of ovulation in dairy cows using $\mathrm{PGF}_{2 \alpha}$ and $\mathrm{GnRH}$. Theriogenology 44:915-923.

Rorie, R. W., T. R. Bilby, and T. D. Lester. 2002. Application of electronic estrus detection technologies to reproductive management of cattle. Theriogenology 57:137-148.

Santos, J. E., W. W. Thatcher, R. C. Chebel, R. L. Cerri, and K. N. Galvao. 2004. The effect of embryonic death rates in cattle on the efficacy of estrus synchronization programs. Anim. Reprod. Sci. 82-83:513-535.

Sartori, R., J. M. Haughian, R. D. Shaver, G. J. Rosa, and M. C. Wiltbank. 2004. Comparison of ovarian function and circulating steroids in estrous cycles of Holstein heifers and lactating cows. J. Dairy Sci. 87:905-920
Schefers, J. M., K. A. Weigel, C. L. Rawson, N. R. Zwald, and N. B. Cook. 2010. Management practices associated with conception rate and service rate of lactating Holstein cpws in large, commercial dairy herds. J. Dairy Sci. 93:1459-1467.

Silva del Rio, N., S. Stewart, P. Rapnicki, Y. M. Chang, and P. M. Fricke. 2007. An observational analysis of twin births, calf sex ratio, and calf mortality in Holstein dairy cattle. J. Dairy Sci. 90:1255-1264.

Souza, A. H., H. Ayres, R. M. Ferreira, and M. C. Wiltbank. 2008. A new presynchronization system (Double-Ovsynch) increases fertility at first postpartum timed AI in lactating dairy cows. Theriogenology 70:208-215.

Sterry, R. A., M. L. Welle, and P. M. Fricke. 2006. Effect of interval from timed artificial insemination to initiation of resynchronization of ovulation on fertility of lactating dairy cows. J. Dairy Sci 89:2099-2109.

Tenhagen, B. A., M. Drillich, R. Surholt, and W. Heuwieser. 2004 Comparison of timed AI after synchronized ovulation to AI at estrus: Reproductive and economic considerations. J. Dairy Sci 87:85-94.

Thatcher, W. W., T. R. Bilby, J. A. Bartolome, F. Silvestre, C. R Staples, and J. E. Santos. 2006. Strategies for improving fertility in the modern dairy cow. Theriogenology 65:30-44.

Thompson, I. M., R. L. Cerri, I. H. Kim, J. A. Green, J. E. Santos, and W. W. Thatcher. 2010. Effects of resynchronization programs on pregnancy per artificial insemination, progesterone, and pregnancy-associated glycoproteins in plasma of lactating dairy cows. J. Dairy Sci. 93:4006-4018.

Thurmond, M. C., and J. P. Picanso. 1990. A surveillance system for bovine abortion. Prev. Vet. Med. 8:41-53

VandeHaar, M., H. Bucholtz, R. Beverly, R. Emery, M. Allen, C Sniffen, and R. Black. 1992. Spartan dairy ration evaluator/balancer. An agricultural integrated management software microcomputer program. CP-012 Version 2.01. CES, AES. Michigan State University, East Lansing.

White, S. L. G. A. Benson, S. P. Washburn, and J. T. Green Jr. 2002. Milk production and economic measures in confinement or pasture systems using seasonally calved Holstein and Jersey cows. J. Dairy Sci. 85:95-104.

Xu, Z. Z., D. J. McKnight, R. Vishwanath, C. J. Pitt, and L. J. Burton. 1998. Estrus detection using radiotelemetry or visual observation and tail painting for dairy cows on pasture. J. Dairy Sci. $81: 2890-2896$. 